\title{
Characteristics of left ventricular filling in coronary artery disease and myocardial ischaemia after dipyridamole provocation
}

\author{
Manjit Shahi, Aleksandar Nadazdin, Rodney A Foale
}

\begin{abstract}
Doppler echocardiographic measurement of transmitral filling velocities seems to be a sensitive marker for resting left ventricular diastolic abnormalities in patients with coronary artery disease. The behaviour of these filling velocities during induced myocardial ischaemia, however, has not been fully studied. Left ventricular filling was assessed by pulsed Doppler ultrasound in 21 patients with angina pectoris and coronary artery disease and in five controls (patients with chest pain but without myocardial ischaemia). High dose dipyridamole infusion $(0.9 \mathrm{mg} / \mathrm{kg}$ over 10 minutes) was used to provoke myocardial ischaemia, which was assessed by symptoms and electrocardiographic ST segment change. Doppler indices of diastolic filling were measured and the results expressed as percentage change from baseline values. Dipyridamole increased the heart rate and reduced systolic blood pressure equally in both groups. In the controls dipyridamole increased the peak filling velocities of both the early and atrial filling waves. In the 12 patients with coronary artery disease who did not develop evidence of myocardial ischaemia, the effect on left ventricular filling velocity resembled that in the controls though the time to peak change was delayed. Six of the nine patients with dipyridamole induced myocardial ischaemia had a significantly reduced maximum changes in early $(+30 \% v$ $+18 \%)$ and atrial $(-0 \cdot 2 \% v+33 \%)$ filling velocities compared with the controls. The remaining three patients had a decrease in early filling velocity $(-20 \%)$ with an associated increase in atrial peak filling velocity $(+21 \%)$.

Dipyridamole increased diastolic filling velocities in the controls. In patients with coronary artery disease there was a variable change in diastolic filling indices which may be attributed either to the degree of myocardial ischaemia or to the different haemodynamic changes occurring during myocardial ischaemia.
\end{abstract}

Transmitral pulsed Doppler ultrasound confirmed resting abnormalities of diastolic function in patients with coronary artery disease. ${ }^{1-3}$
These were a decrease in the peak velocity of the early filling wave together with an increase in peak velocity of the atrial filling wave; changes which in a simple form may be equated with a decrease in left ventricular compliance. This reversal of the normal filling pattern was also seen in those cardiac conditions in which there is evidence of hypertrophy or fibrosis of the left ventricle. ${ }^{4-6}$ In patients with coronary artery disease this finding has been attributed to the presence of left ventricular fibrosis caused by infarction below the thresholds of clinical diagnosis or perhaps subsequent upon recurrent episodes of ischaemia.

During provoked myocardial ischaemia an increase in left ventricular diastolic pressure may lead to a decrease in the transmitral pressure gradient and would therefore be expected to decrease further the peak velocity of the early filling wave. Such changes have been shown in animal models ${ }^{78}$ and also in humans, ${ }^{9-11}$ when during angioplasty of the left anterior descending coronary artery early peak flow velocity fell and atrial velocity increased. In these reports ischaemia was induced by abrupt occlusion of coronary flow by the balloon procedure but it is not known whether these changes occur during demand provoked myocardial ischaemia. This in part is because of the technical difficulties of recording and analysing transmitral Doppler signals during exercise.

As an alternative to exercise dipyridamole can be used to provoke myocardial ischaemia. Dipyridamole is a potent coronary arteriolar vasodilator when it is infused intravenously and it causes myocardial ischaemia possibly by a mechanism of coronary steal. ${ }^{12}$

The aim of this study was first to examine left ventricular filling characteristics by transmitral pulsed Doppler ultrasound during infusion of dipyridamole in controls and in patients with coronary artery disease and secondly to observe the behaviour of left ventricular filling indices in those patients in whom myocardial ischaemia developed.

\section{Patients and methods}

CONTROLS

We studied five controls (mean age 45, range 30-63 years; three men and two women) who presented with chest pain atypical of myocardial ischaemia. In each the resting electrocardiogram was normal and the results of treadmill exercise testing by Bruce's standard protocol showed no ischaemic response. 
Echocardiography with particular reference to left ventricular anatomy was normal. Each patient underwent coronary angiography and left ventriculography at the time of left heart catheterisation to confirm that left ventricular function and the epicardial coronary vessels were normal.

\section{PATIENTS}

We studied 21 patients (mean age 58, range 50-70 years; 13 men and eight women) who presented with chest pain typical of angina pectoris with ST segment depression and chest pain during treadmill exercise testing by Bruce's standard protocol. All patients had normal left ventricular systolic function on echocardiography with no global or regional wall motion abnormality and no left ventricular hypertrophy. Each patient underwent coronary arteriography and left ventriculography and had at least one major stenosis of a coronary vessel $(>70 \%$ reduction in lumen diameter from one or more projections or $>50 \%$ from two or more angiographic projections). All patients had angiographically confirmed normal left ventricular function and no evidence of mitral regurgitation or aortic valve disease. Table 1 shows the patient characteristics of both groups.

The controls were studied without medication. Patients in the study group who were taking $\beta$ blockers and calcium antagonists were treated with sublingual glyceryl trinitrate instead of their normal medication for three days before the study. No drugs were taken on the study day. The study protocol was approved by the hospital ethics committee and written consent was obtained from all patients.

STUDY PROTOCOL

All patients were studied in the supine left lateral position by combined Doppler and cross sectional echocardiography with a $3.5 \mathrm{MHz}$ transducer (General Electric Pass 11). Transmitral flow velocities were recorded by pulsed Doppler from the apical transducer position in a four chamber view with reference to the mitral inflow. From this position the Doppler sample volume was placed at the level of the mitral annulus and parallel to the mitral inflow. We kept the sample volume in a consistent position below the mitral valve apparatus. All recordings were performed during passive held expiration with simultaneous imaging of the left ventricle and lead II of the electrocardiogram.

Recordings of transmitral flow velocities were performed for five minutes to obtain baseline values. Dipyridamole was then infused via an antecubital vein at a dose of 0.6 $\mathrm{mg} / \mathrm{kg}$ over four minutes followed by a dose of $0.3 \mathrm{mg} / \mathrm{kg}$ commencing at eight minutes for a further two minutes. Doppler recordings were taken for approximately 15 seconds every minute during and after the infusion for a total of 20 minutes. Aminophylline (50-250 mg) was given by intravenous injection at the end of the procedure or earlier if chest pain or ST segment depression occurred.

Throughout the 20 minute procedure, automated measurements of heart rate, blood pressure, and 12 lead electrocardiograms were taken every two minutes. In the controls during dipyridamole infusion the mean (1 SD) maximum heart rate increase was $+27(7) \%$ and the maximum fall in systolic blood pressure was $-10(16) \%$ compared with baseline values. In the patients with coronary artery disease the maximum heart rate increase was $+18(10) \%$ and the maximum systolic blood pressure decrease was $-14(10) \%$ during dipyridamole infusion compared with baseline values. Dif-

Table 1 Patient characteristics

\begin{tabular}{|c|c|c|c|c|}
\hline Patient & $\operatorname{Age}(y r)$ & Sex & $C A D$ & Exercise test (time to $S T$ depression (min)) \\
\hline \multicolumn{5}{|l|}{ Controls } \\
\hline $\begin{array}{l}1 \\
2 \\
3 \\
4 \\
5\end{array}$ & $\begin{array}{l}63 \\
30 \\
50 \\
41 \\
43\end{array}$ & $\begin{array}{l}\mathbf{M} \\
\mathbf{M} \\
\mathbf{M} \\
\mathbf{M} \\
\mathbf{F}\end{array}$ & $\begin{array}{l}\text { None } \\
\text { None } \\
\text { None } \\
\text { None } \\
\text { None }\end{array}$ & $\begin{array}{l}\mathbf{N} \\
\mathbf{N} \\
\mathbf{N} \\
\mathbf{N} \\
\mathbf{N}\end{array}$ \\
\hline \multicolumn{5}{|c|}{ Patients with coronary artery disease } \\
\hline $\begin{array}{r}1 \\
2 \\
3 \\
4 \\
5 \\
6 \\
7 \\
8 \\
9 \\
10 \\
11 \\
12 \\
13 \\
14 \\
15 \\
16 \\
17 \\
18 \\
19 \\
20 \\
21\end{array}$ & $\begin{array}{l}52 \\
56 \\
58 \\
54 \\
60 \\
51 \\
55 \\
60 \\
63 \\
51 \\
69 \\
57 \\
68 \\
70 \\
67 \\
50 \\
55 \\
55 \\
54 \\
60 \\
57\end{array}$ & $\begin{array}{l}M \\
M \\
M \\
M \\
M \\
M \\
M \\
M \\
M \\
M \\
F \\
M \\
F \\
F \\
M \\
M \\
M \\
M \\
F \\
M \\
M\end{array}$ & $\begin{array}{l}\text { Cx } \\
\text { LAD + Cx } \\
\text { LAD } \\
\text { LAD } \\
\text { RCA + Cx } \\
\text { LAD } \\
\text { LAD } \\
\text { Cx } \\
\text { LAD } \\
\text { Cx } \\
\text { LAD } \\
\text { LAD } \\
\text { LAD } \\
\text { RCA + } \\
\text { LAD + RCA + Cx } \\
\text { LAD + Cx } \\
\text { LAD + RCA } \\
\text { LAD + RCA } \\
\text { Cx } \\
\text { Cx } \\
\text { RCA + Cx }\end{array}$ & $\begin{array}{r}6 \\
5 \\
6 \\
7 \\
6 \\
8 \\
11 \\
6 \\
4 \\
5 \\
6 \\
4 \\
4 \\
2 \\
2 \\
4 \\
4 \\
5 \\
5 \\
3 \\
6 \\
6 \\
6\end{array}$ \\
\hline
\end{tabular}


values. Differences between the two groups were not statistically significant.

\section{ANALYSIS OF DATA}

The peak flow velocities of both the early and atrial filling waves were measured by one observer using commercial software. The mean of three consecutive cardiac cycles was taken of the early and atrial filling indices for each of the one minute interval recordings. The mean velocities of the five minute preinfusion period were taken as baseline measurements. To allow direct comparison between individuals and between groups we used the percentage change of both early and atrial peak flow velocities from baseline measurements in the analysis of the data.

The 12 lead electrocardiograms were read by one observer and ST segment depression of greater than $1 \mathrm{~mm}$ in the chest or limb leads compared with baseline recordings was regarded as electrocardiographic evidence of myocardial ischaemia.

\section{REPRODUCIBILITY OF MEASUREMENTS}

To determine the reproducibility of the measurements of both peak early and atrial velocities, the original observer analysed 12 one minute interval recordings from 12 of the Doppler studies on two separate occasions. A linear correlation was calculated for the paired observations and the coefficient of variance was used to determine the intraobserver variation.

\section{STATISTICAL ANALYSIS}

All results were expressed as mean (1 SD). We used Student's two tailed $t$ test (unpaired) to test the differences between groups; a $p$ value $<0.05$ was regarded as significant.

Figure 1 Mean percentage change and standard error of both early and atrial filling velocities during the study period. The stippled box is the first infusion of dipyridamole and the hatched box is the second infusion of dipyridamole. PFV A, peak flow velocity of atrial filling wave; PFVE, peak flow velocity of early filling wave.
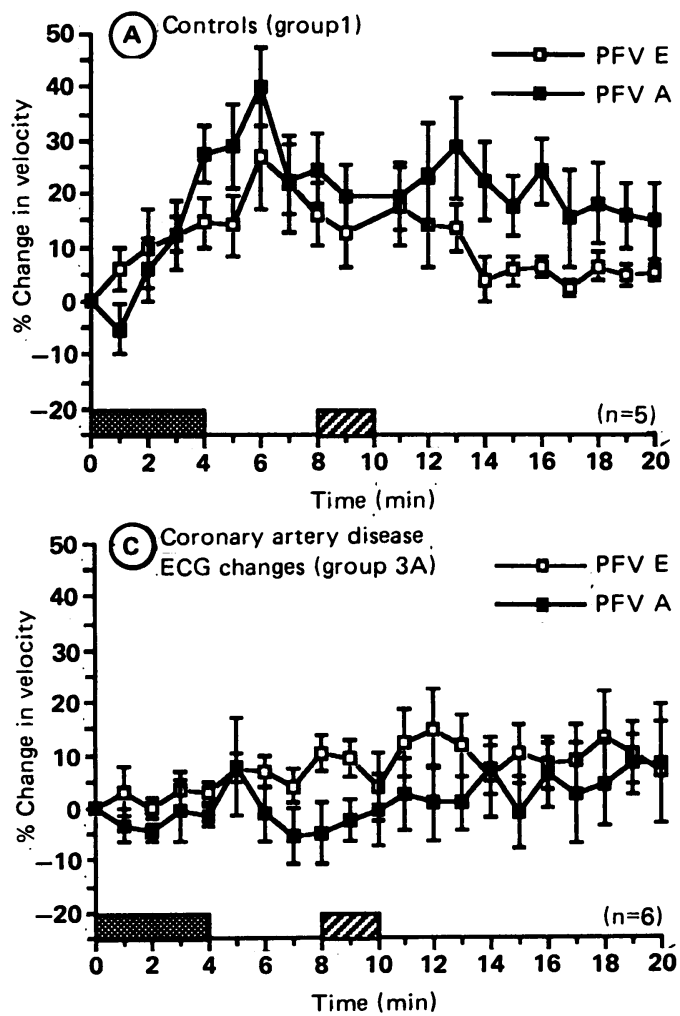

\section{Results}

CLINICAL RESPONSE

None of the controls (group 1) developed chest pain or electrocardiographic change during infusion of dipyridamole. Nor did 12 of the 21 patients with coronary artery disease (group 2). Nine of 21 patients (group 3) developed significant ST segment depression typical of myocardial ischaemia and, of these, six experienced their typical chest pain usually associated with effort. No patient had chest pain without electrocardiographic changes during dipyridamole infusion.

LEFT VENTRICULAR FILLING CHARACTERISTICS The results of the Doppler study were analysed for left ventricular filling changes during infusion of dipyridamole. Figure $1 \mathrm{~A}$ shows the effect of dipyridamole on left ventricular filling in group 1. In general there was an initial rapid increase in both peak early and atrial filling velocities with a maximum change in both velocities shortly after the first infusion of dipyridamole. The peak velocities then gradually decreased but did not reach baseline values at the end of the study period.

In most of the patients in group 2 the changes in both early and atrial filling velocities were similar to the changes in group 1. However, filling velocity changes reached a maximum after the second infusion of dipyridamole (Fig 1B). Patients in group 3 had two different left ventricular filling characteristics. Six of the nine patients (group 3A) showed a reduced maximum change in both early and atrial filling velocities compared with most of the patients in groups 1 and 2 (Fig $1 \mathrm{C}$ ). The remaining three patients (group 3B) had a decrease in maximum change in early filling velocity accompanied by an increase in atrial filling velocity (Fig 1D).
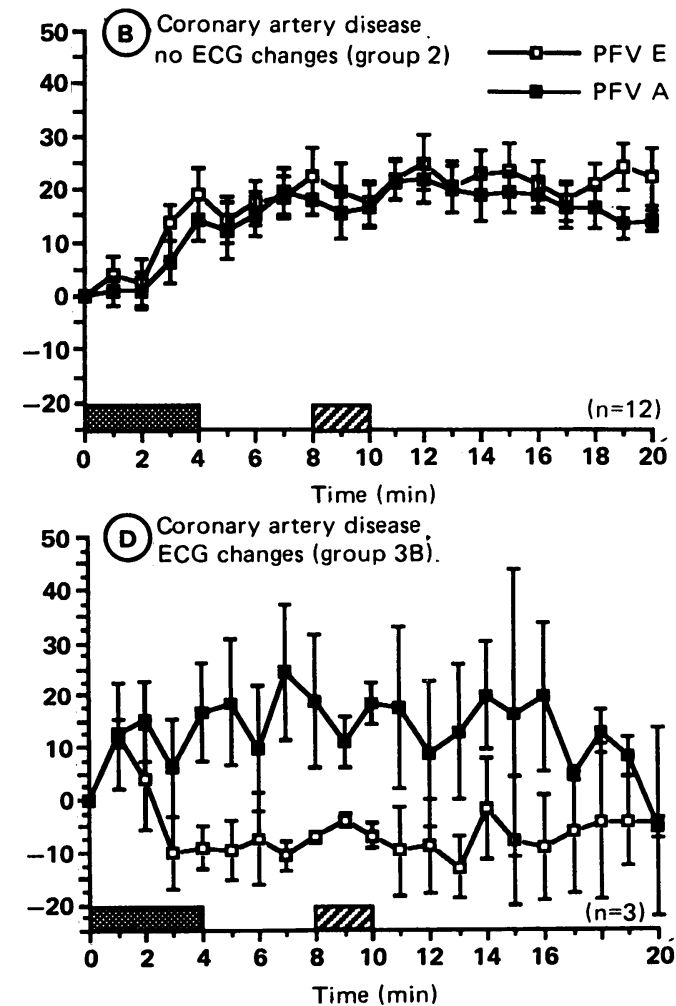
Table 2 Maximum filling velocities

\begin{tabular}{|c|c|c|c|c|c|c|c|c|c|c|c|}
\hline & $\% H R$ & $\% B P$ & $E_{b}(\mathrm{~cm} / \mathrm{s})$ & $E_{m}(\mathrm{~cm} / \mathrm{s})$ & $\% E$ & $A_{b}(\mathrm{~cm} / \mathrm{s})$ & $A_{m}(\mathrm{~cm} / \mathrm{s})$ & $\% A$ & $E / A_{b}$ & $E / A_{m}$ & $\% E / A$ \\
\hline \multicolumn{12}{|c|}{ Group 1: } \\
\hline $\begin{array}{l}1 \\
2 \\
3 \\
4 \\
5\end{array}$ & $\begin{array}{l}19 \\
28 \\
24 \\
25 \\
37\end{array}$ & $\begin{array}{r}39 \\
6 \\
0 \\
3 \\
4\end{array}$ & $\begin{array}{l}31 \\
70 \\
45 \\
60 \\
59\end{array}$ & $\begin{array}{l}48 \\
88 \\
51 \\
76 \\
77\end{array}$ & $\begin{array}{l}55 \\
25 \\
15 \\
26 \\
30\end{array}$ & $\begin{array}{l}56 \\
45 \\
67 \\
78 \\
60\end{array}$ & $\begin{array}{l}72 \\
79 \\
80 \\
85 \\
89\end{array}$ & $\begin{array}{r}30 \\
75 \\
20 \\
9 \\
29\end{array}$ & $\begin{array}{l}0.56 \\
1.56 \\
0.67 \\
0.78 \\
0.98\end{array}$ & $\begin{array}{l}0.67 \\
1.11 \\
0.64 \\
0.9 \\
0.78\end{array}$ & $\begin{array}{r}18 \\
41 \\
-4 \\
15 \\
20\end{array}$ \\
\hline \multicolumn{12}{|c|}{ Group 2: } \\
\hline $\begin{array}{r}1 \\
2 \\
3 \\
4 \\
5 \\
6 \\
7 \\
8 \\
9 \\
10 \\
11 \\
12\end{array}$ & $\begin{array}{r}40 \\
21 \\
11 \\
21 \\
7 \\
35 \\
12 \\
12 \\
15 \\
17 \\
25 \\
32\end{array}$ & $\begin{array}{r}17 \\
16 \\
6 \\
0 \\
13 \\
0 \\
36 \\
3 \\
0 \\
19 \\
11 \\
10\end{array}$ & $\begin{array}{l}72 \\
46 \\
28 \\
43 \\
50 \\
50 \\
46 \\
50 \\
38 \\
58 \\
37 \\
62\end{array}$ & $\begin{array}{l}91 \\
68 \\
35 \\
67 \\
62 \\
79 \\
62 \\
55 \\
59 \\
69 \\
59 \\
78\end{array}$ & $\begin{array}{r}27 \\
47 \\
24 \\
57 \\
24 \\
59 \\
34 \\
8 \\
54 \\
20 \\
62 \\
26\end{array}$ & $\begin{array}{l}63 \\
58 \\
44 \\
56 \\
66 \\
56 \\
59 \\
63 \\
49 \\
72 \\
77 \\
59\end{array}$ & $\begin{array}{l}82 \\
81 \\
56 \\
84 \\
77 \\
80 \\
71 \\
58 \\
59 \\
75 \\
98 \\
79\end{array}$ & $\begin{array}{r}29 \\
39 \\
27 \\
49 \\
16 \\
42 \\
22 \\
-17 \\
21 \\
3 \\
27 \\
34\end{array}$ & $\begin{array}{l}1.13 \\
0.73 \\
0.62 \\
0.76 \\
0.75 \\
0.89 \\
0.78 \\
0.81 \\
0.78 \\
0.8 \\
0.47 \\
1.04\end{array}$ & $\begin{array}{l}1.0 \\
0.84 \\
0.62 \\
0.8 \\
0.8 \\
1.0 \\
0.87 \\
0.95 \\
1.0 \\
0.93 \\
0.6 \\
0.98\end{array}$ & $\begin{array}{r}-11 \\
15 \\
0 \\
5 \\
6 \\
12 \\
11 \\
17 \\
28 \\
16 \\
28 \\
-5\end{array}$ \\
\hline \multicolumn{12}{|c|}{ Group 3A: } \\
\hline $\begin{array}{l}1 \\
2 \\
3 \\
4 \\
5 \\
6\end{array}$ & $\begin{array}{r}19 \\
28 \\
21 \\
2 \\
18 \\
11\end{array}$ & $\begin{array}{l}22 \\
13 \\
36 \\
24 \\
12 \\
24\end{array}$ & $\begin{array}{l}45 \\
47 \\
47 \\
45 \\
41 \\
51\end{array}$ & $\begin{array}{l}50 \\
52 \\
64 \\
58 \\
47 \\
56\end{array}$ & $\begin{array}{r}11 \\
10 \\
37 \\
29 \\
9 \\
9\end{array}$ & $\begin{array}{l}37 \\
85 \\
54 \\
88 \\
74 \\
62\end{array}$ & $\begin{array}{l}33 \\
81 \\
66 \\
82 \\
73 \\
62\end{array}$ & $\begin{array}{r}-11 \\
-4 \\
23 \\
-8 \\
-1 \\
0\end{array}$ & $\begin{array}{l}1.2 \\
0.56 \\
0.87 \\
0.55 \\
0.56 \\
0.83\end{array}$ & $\begin{array}{l}1.5 \\
0.64 \\
0.97 \\
0.66 \\
0.65 \\
0.93\end{array}$ & $\begin{array}{l}25 \\
14 \\
11 \\
20 \\
16 \\
12\end{array}$ \\
\hline \multicolumn{12}{|c|}{ Group 3B: } \\
\hline $\begin{array}{l}1 \\
2 \\
3\end{array}$ & $\begin{array}{r}5 \\
21 \\
24\end{array}$ & $\begin{array}{r}6 \\
12 \\
13\end{array}$ & $\begin{array}{l}78 \\
79 \\
92\end{array}$ & $\begin{array}{l}58 \\
63 \\
79\end{array}$ & $\begin{array}{l}-25 \\
-20 \\
-14\end{array}$ & $\begin{array}{l}52 \\
31 \\
36\end{array}$ & $\begin{array}{l}54 \\
36 \\
52\end{array}$ & $\begin{array}{r}4 \\
16 \\
44\end{array}$ & $\begin{array}{l}1.52 \\
2.55 \\
2.54\end{array}$ & $\begin{array}{l}1.08 \\
1.74 \\
1.52\end{array}$ & $\begin{array}{l}-29 \\
-32 \\
-40\end{array}$ \\
\hline
\end{tabular}

$\% \mathrm{HR}$, maximum percentage increase in heart rate; \% BP, maximum percentage decrease in systolic blood pressure; $\mathrm{E}_{\mathrm{b}}$, baseline early filling velocity; $A_{b}$, baseline atrial filling velocity; $E / A_{b}$, baseline ratio of early and atrial filling velocities; $E_{m}$, maximum early
filling velocity; $A_{m}$, maximum atrial filling velocity; $E / A_{m}$, maximum ratio of early and atrial filling velocities; $\%, \%, \%, E / A$, filling velocity; $A_{m}$, maximum atrial
percentage change from baseline.

\section{INTRAOBSERVER VARIABILITY OF DOPPLER MEASUREMENTS}

There was a strong linear correlation for repeated measurements of peak early and filling velocities $\left(\mathrm{r}^{2}=0.99\right.$ and $\mathrm{r}^{2}=0.98$ respectively). The intraobserver variation for repeated measurements gave a coefficient of variance of $1.2 \%$ for peak early velocity and $1.4 \%$ for peak atrial velocity.

\section{DOPPLER ANALYSIS AND CHANGES DURING MYOCARDIAL ISCHAEMIA}

Table 2 shows the individual values for the haemodynamic changes during dipyridamole infusion and the early and atrial filling velocities and the E/A ratio at baseline and when the maximum early filling velocity was achieved in the study population.

The percentage change in early filling velocity in group 1 and group 2 patients $(30.2$ (15) and $36 \cdot 8$ (18) respectively) was significantly higher than in group $3 \mathrm{~A}$ patients (18.5 (12); $\mathrm{p}<0.05)$ and all these values were significantly different from the value in group 3B $(-19 \cdot 7$ $(5.5) ; p<0.01)$. The percentage increase in atrial filling was similar in group 1, group 2, and group $3 B$ patients $(32.6(25), 25.2(15.9)$, and $21.3(20.5))$ and all were higher than in the group $3 \mathrm{~A}$ patients $(-0.2(12 \cdot 1) ; \mathrm{p}<0.05)$. The percentage change in $E / A$ ratio in group $3 B$ $(-33.3(5 \cdot 7)$ was significantly lower than in group 1, group 2, and group $3 \mathrm{~A}$ patients (18 (16), $10.2(11.9)$, and 16.3 (5.3); p < 0.01) (fig 2).

\section{Discussion}

Left ventricular filling is a complex process that is governed by several interdependent variables. Theoretically it is regulated by the atrioventricular pressure difference generated by the elastic recoil of the left ventricle in diastole. The use of transmitral Doppler ultrasound to measure peak filling velocities, which are directly proportional to the atrioventricular difference, therefore allows noninvasive assessment of left ventricular filling. ${ }^{13}$ The atrioventricular pressure difference, however, is influenced by left atrial pressure, rate of relaxation, left ventricular end systolic volume, and left ventricular and left atrial compliance ${ }^{14-16}$ and therefore its usefulness as an index of diastolic function is thus dependent on the interpretation of these factors.

We studied the effects of dipyridamole infusion on left ventricular filling in controls and patients with coronary artery disease. Dipyridamole is known significantly to increase coronary blood flow and to provoke myocardial ischaemia mainly by a mechanism of coronary steal but also by slightly increasing myocardial oxygen consumption. ${ }^{12}$ In the controls (group 1) the increased coronary flow induced by dipyridamole increased both early and atrial filling velocities. The concept that increased left ventricular filling velocities correlate to an increase in coronary flow accords with previous studies that showed similar favourable changes when coronary flow was improved to normal values by either aortocoronary artery bypass graft $s^{17}$ or successful coronary angioplasty. ${ }^{18}$

The increase in early filling velocities consequent upon increased coronary flow in the controls can be explained by the haemodynamic events that take place during dipyridamole infusion. Picano et al showed that 

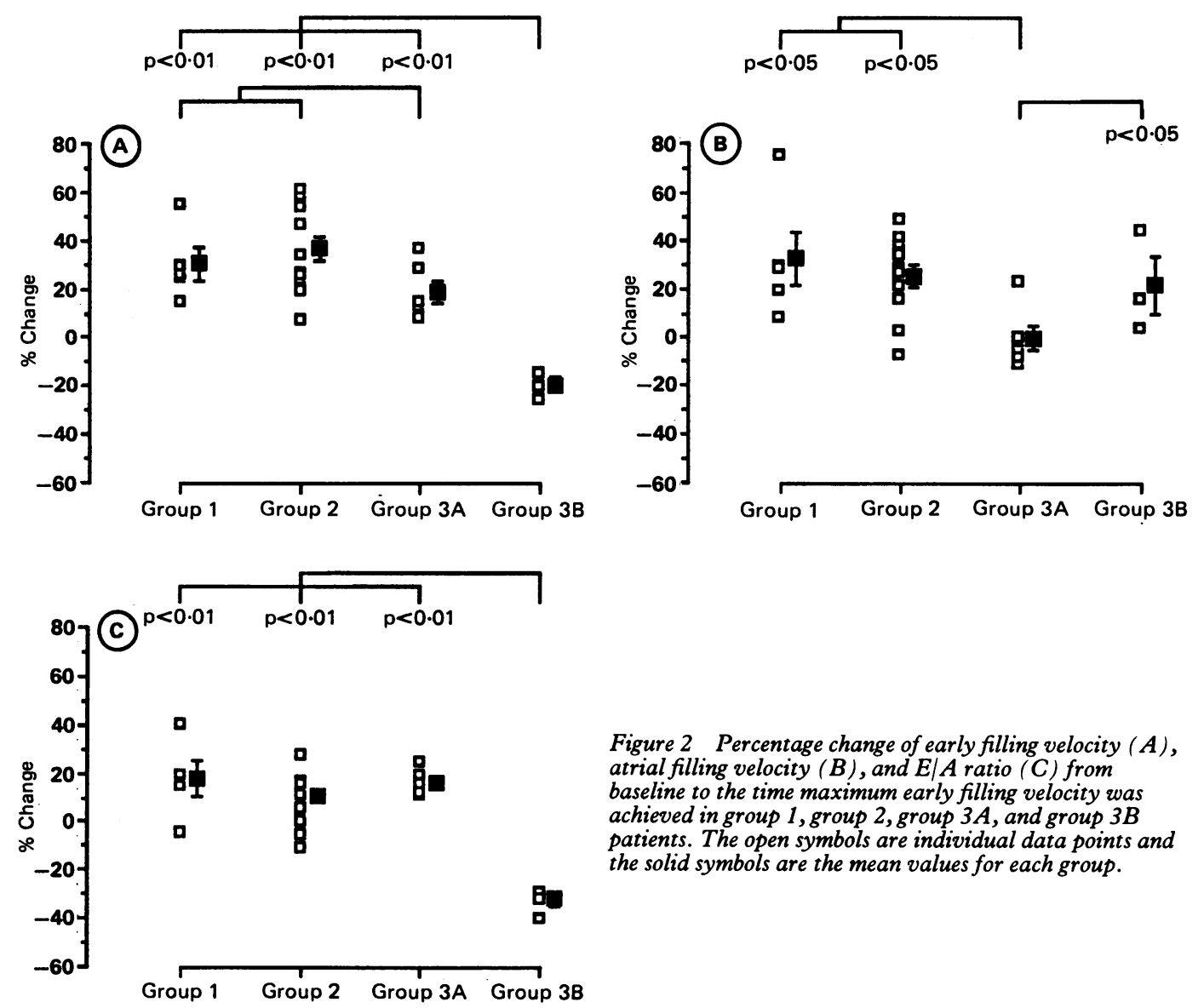

Figure 2 Percentage change of early filling velocity $(A)$, atrial filling velocity $(B)$, and $E / A$ ratio $(C)$ from baseline to the time maximum early filling velocity was achieved in group 1 , group 2 , group $3 A$, and group $3 B$ patients. The open symbols are individual data points and the solid symbols are the mean values for each group.

during dipyridamole infusion both left ventricular contractility (positive $\mathrm{dP} / \mathrm{dt}$ ) and left ventricular relaxation (negative $\mathrm{dP} / \mathrm{dt}$ ) are increased whereas right ventricular pressures remain the same. ${ }^{19}$ The overall effect is that left ventricular relaxation is faster and more complete; this enhances the atrioventricular pressure difference and consequently increases the early peak filling velocity.

In patients with coronary artery disease the left ventricular filling response fell into three broadly based categories. These categories represented a continuum from a normal response to a severe disturbance of left ventricular filling. The first resembled that in the controls and occurred in patients who did not develop electrocardiographic changes of myocardial ischaemia (group 2). In these patients there was a large increase in both early and atrial filling velocities. The second response occurred in six of the nine patients (group 3A) with dipyridamole provoked myocardial ischaemia. In this group most of the patients had a reduced but still positive change in maximum early filling velocity compared with the controls. This finding might suggest that these patients initially increased their coronary blood flow and that after the onset of myocardial ischaemia a decrease in the atrioventricular pressure difference limited any further rise in the early peak flow velocity. This hypothesis is supported by earlier studies which showed that during myocardial ischaemia the rate of relaxation (tau) is prolonged and because this also affects the atrioventricular pressure difference, peak flow velocity falls. ${ }^{21} 21$ The reduced atrial filling

velocities in these patients may be due to a failure of the normal atrial compensatory mechanism to maintain stroke volume in the presence of a reduced early peak filling rate and therefore possibly a reflection of decreased left atrial or left ventricular compliance or both.

The third response was that observed in patients of group 3B, in whom the early filling velocity decreased despite a theoretical increase in coronary flow during dipyridamole infusion. These patients seem to represent a group who develop more pronounced myocardial ischaemia and have a similar response to that seen when coronary vessels are occluded during balloon angioplasty. ${ }^{9}$

In the study from Lattanzi and coworkers similar changes were seen in transmitral flow velocities in patients with coronary artery disease who developed regional wall motion abnormalities during infusion of dipyridamole. ${ }^{22}$ However, using a lower dose of dipyridamole, Grayburn et al were unable to record any differences in left ventricular filling for patients with coronary artery disease despite the documentation of dipyridamole induced thallium defects. ${ }^{23}$ This group, however, only obtained one measurement of the transmitral flow velocities at an arbitrary time after dipyridamole infusion.

Despite the significant differences in left ventricular filling velocities between patients who did develop myocardial ischaemia and those who did not, there was some overlap between these groups. This implies that in individual patients, changes in left ventricular filling velocities during dipyridamole infusion could 
not with certainty predict whether myocardial ischaemia had occurred. If an earlier and more sensitive marker of myocardial ischaemia such as echocardiographic assessment of regional wall motion ${ }^{24}{ }^{25}$ had been used, however, it is possible that myocardial ischaemia may have occurred in some of the patients in whom ST segment depression did not develop (patients 8 and 10 , group 2 , table 2 ) but who had similar left ventricular filling velocities to patients who did (group 3A).

\section{LIMITATIONS OF THE STUDY}

Though no significant difference was seen in the haemodynamic changes produced by the infusion of dipyridamole in the two study groups, there was considerable variation between the subgroups particularly with respect to the blood pressure response and this might account for the observed changes in left ventricular filling. The effects of an acute reduction in blood pressure on left ventricular filling in patients with coronary artery disease has not been studied but indirect evidence from several investigators showed that reducing blood pressure in hypertensive patients ${ }^{26}$ or acutely increasing blood pressure in normal individuals ${ }^{27}$ had no effect on Doppler indices of left ventricular filling.

Another limitation of the study was that further valuable information derived from both the early and atrial filling waveforms could not be accurately analysed because the increase in heart rate induced by dipyridamole caused the waveforms to merge. The flow velocity integral gives a reliable estimate of cardiac output ${ }^{28}$ and would have given valuable information in our patients on whether total cardiac output or the relative proportions of early and late transmitral volume flow were altered by dipyridamole infusion. The deceleration time of the early filling wave is known to be increased in patients with coronary artery disease $\mathrm{e}^{29}$ and may have further increased during myocardial ischaemia. However, even with these limitations we obtained useful information from peak flow velocities of both early and atrial filling waves during dipyridamole infusion which could not be obtained during exercise because the heart rates required to provoke myocardial ischaemia would have precluded even the measurements of peak flow velocities.

In conclusion, our study showed that dipyridamole increased the left ventricular filling velocities in the controls. In those patients who developed myocardial ischaemia, two distinct responses were seen which may be attributed either to the degree of myocardial ischaemia or to different haemodynamic changes occurring during myocardial ischaemia.

1 Fugii J, Yazaki Y, Sawada H, Aizawa T, Watanabe H, Kato $K$. Noninvasive assessment of left and right ventricular filling in myocardial infarction with a 2 dimensional Doppler echocardiographic method. J Am Coll Cardiol 1985;5:1155-60.

2 Kitabatake A, Inoue M, Asao M, et al. Transmitral blood flow reflecting diastolic behaviour of the left ventricle in health and disease: a study by pulsed Doppler technique. Jpn Circ J 1982;46:92-102.

3 Lin SL, Tak T, Gamage N, Kawanishi DT, McKay CR. Assessment of left ventricular diastolic function in coronary artery disease by Doppler ultrasound [Abstract]. Circulation 1986;74(suppl II):47.

4 Dianzumba SB, DiPette DJ, Cornman C, Weber E, Joyner CR. Left ventricular filling characteristics in mild untreated hypertension. Hypertension 1986;8:1156-60.

5 Takenaka K, Sakamoto T, Amano K, et al. Left ventricular filling determined by Doppler echocardiography in filling determined by Doppler echocardiograp

6 Bryg RJ, Pearson AC, Williams GA, Labovitz A. Left ventricular systolic and diastolic flow abnormalities deterventricular systolic and diastolic flow abnormalities deter-
mined by Doppler echocardiography in obstructive mined by Doppler echocardiography in obstructive hypertrophic

7 Fisher DC, Voyles WF, Sikes W, Greene ER. Left ventricular filling patterns during ischaemia: an echo/Doppler study in open chest dogs [Abstract]. J Am Coll Cardiol 1985;5:426A.

8 Ridner ML, Courtois MR, Perez JE, Barzilia B, Ludbrook PA. Alterations in Doppler indexes of diastolic function following coronary artery reperfusion [Abstract]. Circulation 1986;74(suppl II):47.

9 Bruyne B, Lerch R, Meier B, Schlaepfer H, Gabathuler J, Rutishauser W. Doppler assessment of left ventricular diastolic filling during brief coronary occlusion. Am Heart diastolic filling during

10 Labovitz A Lewen M, Kern M, Vandormael M, Habermehl $\mathrm{K}$. Temporal relationship of left ventricular systolic and diastolic dysfunction during percutaneous transand diastolic dysfunction during percutaneous trans-
luminal coronary angioplasty [Abstract]. Circulation luminal coronary an
$1986 ; 74$ (suppl II): 358

11 Henderson M, Schwartz L, Aldridge HE, Prieur T, Rakowski $H$. Doppler quantitation of diastolic function during PTCA induced ischaemia [Abstract]. Circulation 1986;74(suppl II):357.

12 Picano E. Dipyridamole echocardiography test: historical background and physiological basis. Eur Heart $J$ 1989; 10:365-76.

13 Courtois M, Kovacs SJ, Ludbrook PA. Transmitral pressure-flow velocity relation. Circulation 1988;78:661-71.

14 Ishida Y, Meisner JS, Tsujioka K, et al. Left ventricular filling dynamics: influence of left ventricular relaxation and left atrial pressure. Circulation 1986;68:187-96.

15 Yellin EL, Sonnenblick EH, Frater RWM. Dynamic determinants of left ventricular filling: an overview. In: Baan J, minants of left ventricular filling: an overview. In: Baan J,
Arntzenius AC, Yellin EL, et al, eds. Cardiac dynamics. Arntzenius AC, Yellin EL, et al, eds. Cardiac
The Hague: Martinus Nijhoff, 1980:145-58.

16 Weisfeldt ML, Frederiksen JW, Yin FCP, Weiss JL. Evidence of incomplete left ventricular relaxation in the dog. J Clin Invest 1978;62:1296-302.

17 Lawson W, Seifert F, Anagnostopoulos C, Hills DJ, Swinford RD, Cohn PF. Effect of coronary artery bypass grafting on left ventricular diastolic function. $A m J$ Cardiol 1988;61:283-7.

18 Bonow RO, Kent KM, Rosing DR, et al. Improved left ventricular diastolic filling in patients with coronary artery disease after percutaneous transluminal coronary angioplasty. Circulation 1982;66:1159-67.

19 Picano E, Simonetti I, Carpeggiani C, et al. Regional and global biventricular function during dipyridamole stress testing. Am J Cardiol 1989;63:429-32.

20 Mann T, Goldberg S, Mudge GH, Grossman W. Factors contributing to altered left ventricular diastolic properties contributing to altered left ventricular diastolic proper

21 Dawson JR, Gibson DG. Left ventricular filling and early diastolic function at rest and during angina in patients with coronary artery disease. Br Heart J 1989;61:248-57.

22 Lattanzi F, Picano E, Masini M, DePrisco F, Distante A, L'Abbate A. Transmitral flow changes during dipyridamole induced ischaemia. Chest 1989;95:1037-42.

23 Grayburn PA, Popma JJ, Pryor SL, Walker BS, Simon TR, Smitherman TC. Comparison of dipyridamole-Doppler echocardiography to thallium-201 imaging and quantitative coronary arteriography in the assessment of coronary artery disease. Am J Cardiol 1989;63:1315-20.

24 Picano E, Lattanzi F, Masini M, Distante A, L'Abbate A. Different degrees of ischaemic threshold stratified by the dipyridamole echocardiography test. $A m J$ Cardiol 1987;59:71-3.

25 Margonato A, Chierchia S, Cianflone D, et al. Limitations of dipyridamole echocardiography in effort angina pectoris. Am J Cardiol 1987;59:225-30.

26 Shahi M, Thom S, Poulter N, Sever PS, Foale RA. Does reduction in blood pressure improve left ventricular filling in the hypertensive hea

27 Smith SA, Stoner JE, Russell AE, Sheppard JM, Aylward PE. Transmitral velocities measured by pulsed Doppler in healthy volunteers: effects of acute changes in blood pressure and heart rate. Br Heart $J$ 1989;61:344-7.

28 Lewis JF, Kuo LC, Nelson JG, Limacher MC, Quinones MA. Pulsed Doppler echocardiographic determination of stroke volume and cardiac output: clinical validation of two new methods using the apical window. Circulation 1984;70:425-31

29 Nishumura RA, Abel MD, Hatle LK, Tajik AJ. Assessment of diastolic function of the heart: background and current applications of Doppler echocardiography. Mayo Clin Proc 1989;64:181-204. 